\title{
Correction to: Cytotoxic and genotoxic assessment of tungsten oxide nanoparticles in Allium cepa cells by Allium ana-telophase and comet assays
}

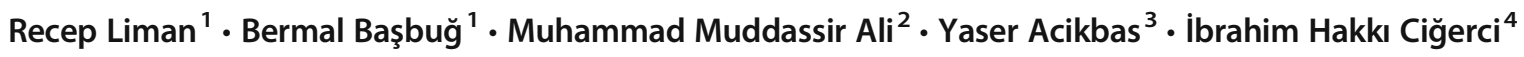 \\ Published online: 18 January 2021 \\ (C) Institute of Plant Genetics, Polish Academy of Sciences, Poznan 2021
}

Correction to: Journal of Applied Genetics (2021) https://doi.org/10.1007/s13353-020-00608-x

The original article was published with an error. The content of Tables 2 and 3 incorrectly interchanged during the Publication.

The original article has been corrected.

Publisher's note Springer Nature remains neutral with regard to jurisdictional claims in published maps and institutional affiliations.

The online version of the original article can be found at https://doi.org/ 10.1007/s13353-020-00608-x

\section{Muhammad Muddassir Ali} muddassir.ali@uvas.edu.pk

1 Faculty of Arts and Sciences, Molecular Biology and Genetics Department, Usak University, 64300 Usak, Turkey

2 Institute of Biochemistry and Biotechnology, University of Veterinary and Animal Sciences, Syed Abdul Qadir Jilani Road (Outfall Road), Lahore 54000, Pakistan

3 Faculty of Engineering, Materials Science and Nanotechnology Engineering Department, Usak University, 64200 Usak, Turkey

4 Faculty of Arts and Sciences, Molecular Biology and Genetics Department, Afyon Kocatepe University,

03200 Afyonkarahisar, Turkey 